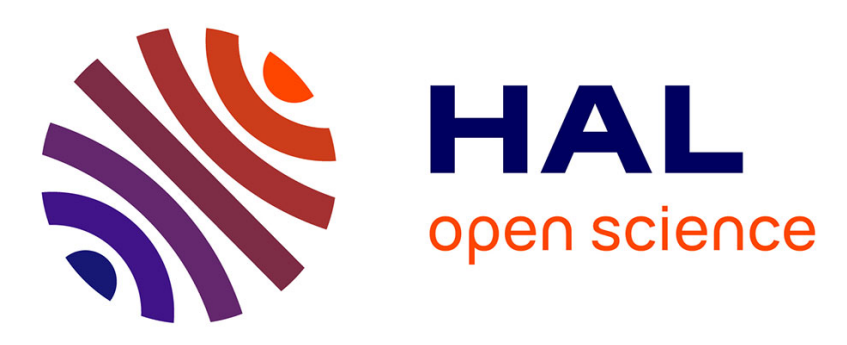

\title{
Introduction au dossier: les phénomènes de hiérarchisation et leurs traitements dans les sociétés anciennes et actuelles
}

Steeve Gentner, Thomas Hutin, Šárka Válečková

\section{To cite this version:}

Steeve Gentner, Thomas Hutin, Šárka Válečková. Introduction au dossier : les phénomènes de hiérarchisation et leurs traitements dans les sociétés anciennes et actuelles. Archimède: archéologie et histoire ancienne, 2017, 4, pp.155-157. halshs-01589310

\section{HAL Id: halshs-01589310 \\ https://shs.hal.science/halshs-01589310}

Submitted on 18 Sep 2017

HAL is a multi-disciplinary open access archive for the deposit and dissemination of scientific research documents, whether they are published or not. The documents may come from teaching and research institutions in France or abroad, or from public or private research centers.
L'archive ouverte pluridisciplinaire HAL, est destinée au dépôt et à la diffusion de documents scientifiques de niveau recherche, publiés ou non, émanant des établissements d'enseignement et de recherche français ou étrangers, des laboratoires publics ou privés. 


\section{ARCHIMÈDE N4}

\section{DOSSIER THÉMATIQUE 1 : NOMMER LES « ORIENTAUX » DANS L’ANTIQUITÉ}

\section{DOSSIER THÉMATIQUE 2 : PRYTANÉE ET REGIA}

\section{ACTUALITÉ DE LA RECHERCHE : DYNAMIQUES HUMAINES ANCIENNES}

\section{Steeve GENTNER, Thomas HUTIN et Šárka VÁLEČKOVÁ}

Introduction au dossier : les phénomènes de hiérarchisation et leurs traitements dans les sociétés anciennes et actuelles

158 Pierre LE ROUX et Alain BEYRAND

D'un drame à un don. Hommage à Louise Beyrand et Olivier Toussaint

162 Alexandra CONY

Hiérarchie de I'habitat rural à la fin de l'âge du Fer. L'importance de définir les critères de hiérarchisation en fonction de l'environnement archéologique

174 Christine HUE-ARCÉ

Hiérarchies socio-professionnelles et violence interpersonnelle dans l'Égypte du Nouvel Empire et d'époque hellénistique

184 Aurélien LANDON

La hiérarchie dans le recrutement militaire : I'exemple épigraphique du recrutement des tribuns militaires durant le Principat d'Auguste

196 Sophie TRIERWEILER

La conception et l'expression d'un droit structuré dans la société homérique à travers les notions de themis et dikê

207 François FAVORY

Organisation et hiérarchisation de l'habitat antique : l'expérience d'Archaeomedes et d'ArchaeDyn

VARIA

216 Jean-Claude LACAM

Prestota Serfia Serfer Martier, la déesse immobile (étude ombrienne, III ${ }^{\mathrm{e}}-\mathrm{II} \mathrm{e}^{\mathrm{e}}$ siècles av. J.-C.)

229 Pierre SCHNEIDER

Des Indiens dans les armées hellénistiques : une autre rencontre entre la Grèce et I'Inde

\section{LA CHRONIQUE D’ARCHIMÈDE}

236 Frédéric COLIN (éd.)

La Chronique d'Archimède. Bilan des activités scientifiques 2016-2017 de I'unité mixte

\section{@creative}




\section{ACTUALITÉ DE LA RECHERCHE DYNAMIQUES HUMAINES ANCIENNES}

\section{INTRODUCTION AU DOSSIER \\ LES PHÉNOMĖNES DE HIÉRARCHISATION ET LEURS TRAITEMENTS DANS LES SOCIÉTÉS ANCIENNES ET ACTUELLES}

\section{Steeve GENTNER}

Doctorant en Archéologie

Université de Strasbourg

UMR 7044 Archimède

steeve.gentner@etu.unistra.fr

\author{
Thomas HUTIN \\ Doctorant en Archéologie \\ Université de Strasbourg \\ UMR 7044 Archimède \\ thomas.hutin01@gmail.com
}

\author{
Šárka VÁLEČKOVÁ \\ Doctorante en Archéologie \\ Université de Strasbourg \\ UMR 7044 Archimède \\ sarka.valeckova@etu.unistra.fr
}

Si la tendance actuelle laisse imaginer que la hiérarchie ne renvoie qu'à des considérations sociales, son champ d'application est en réalité bien plus extensif. Dans ce dossier, il a donc été choisi de retenir le sens générique qui peut être attribué à ce terme, celui que I'on peut trouver dans toute encyclopédie. La hiérarchie peut se définir comme une organisation verticale d'un ensemble donné selon un ou des critères établis en amont. Ce terme très polysémique, parfois ambigu se retrouve de manière récurrente dans le domaine des sciences humaines et sociales.

C'est ainsi que les articles réunis dans ce dossier constituent les actes d'une table ronde qui s'est tenue à la Maison Interuniversitaire des Sciences de I'Homme-Alsace (MISHA) à Strasbourg le 19 octobre 2015, sur la « Hiérarchie et Hiérarchisation dans les sociétés anciennes et actuelles ». Cette rencontre, organisée dans le cadre du « Projet de Jeunes Chercheurs de I'UMR 7044 - Archimède », venait clore une série de 3 Workshops dont la problématique avait su fédérer de nombreux archéologues, historiens et ethnologues. C'est à ce titre que le thème abordé se voulait volontairement large, permettant de combiner des données disparates et des avis épars, favorisant ainsi le contact entre chercheurs issus de différents horizons disciplinaires.

C'est dans un premier temps le monde rural, et plus exactement la distinction du rang hiérarchique d'un habitat dans son terroir, qui fait l'objet d'un éclairage par Alexandra Cony. Sa contribution, dans la lignée des travaux de F. Malrain, démontre tout I'intérêt d'établir des grilles d'analyse modulables et de définir une multiplicité de critères hiérarchiques pour un seul et même individu. Il en découle l'établissement de cinq groupes d'indices, 
allant de l'organisation et de la structuration morphologique du site aux activités de production identifiées sur ce dernier. À noter que cette grille inédite donne la part belle aux occupants eux-mêmes du site étudié. C'est ainsi qu'un éclairage est donné à la fois sur la qualité de consommation des habitants au travers des restes de l'alimentation carnée et des mobiliers d'importations, mais aussi, pour reprendre les termes de l'auteure «la notoriété des habitants », par le biais d'activités symboliques et guerrières parfois plus difficilement identifiables. Cet article ouvre directement la voie à de nouvelles recherches; d'autres focales géographiques, régionales et micro-régionales, pourraient, par le biais de multiples critères, mettre en évidence la variabilité hiérarchique de différents terroirs ou encore le rang d'un habitat dans un réseau plus global.

Au travers des textes néo-égyptiens et démotiques, Christine Hue-Arcé nous plonge dans I'Égypte du Nouvel Empire et d'époque hellénistique et plus particulièrement dans les actes de violence interpersonnelle qui peuvent être identifiés dans le cadre d'une hiérarchie sociale ou professionnelle. Plus globalement, l'auteure s'interroge sur la perception par la société de ces violences et les contextes sociaux dans lesquels elles interviennent. Les corrélations et distinctions qu'elle observe entre les deux époques choisies apportent un éclairage comparatiste essentiel à la mise en exergue de phénomènes de continuité, ou au contraire de ruptures dans les bastonnades qu'un supérieur peut infliger à son subordonné. Que ce soit pour le Nouvel Empire ou l'époque hellénistique, il apparaît que ces violences semblent socialement acceptées, si tant est qu'elles soient pondérées, mesurées, voire déconseillées envers une victime en situation de vulnérabilité. Si globalement les actes de violence semblent donc acceptés, l'acharnement et les abus sont déconseillés, si ce n'est dans certains cas punis. Si les points de convergence sont nombreux, une différence de taille existe. Comme le démontre I'auteure, la rupture qui ressort de son étude réside dans la divergence d'appréciation des actes de violence selon la catégorie littéraire abordée. C'est à ce titre que l'évolution de l'insertion sociale du scribe dans la société pourrait être l'une des réponses à ce contraste.

Après les tensions du milieu et de la fin $\mathrm{du}^{\mathrm{er}}$ siècle av. J.-C. et au lendemain de l'avènement du Principat d'Auguste, I'ossature institutionnelle militaire se voit profondément modifiée. C'est ainsi qu'Aurélien Landon propose, par le biais des sources épigraphiques, d'analyser les différents rapports hiérarchiques que le recrutement des tribuns militaires peut révéler durant la période qui suit les années 27 av. J.-C. L'étude exhaustive des inscriptions mentionnant les tribuns militaires du Principat d'Auguste lui a permis de démontrer que trois grandes inégalités hiérarchiques sont à l'œuvre dans cet enrôlement. Tout d'abord, il apparaît que la pyramide sociale romaine se reflète au sein même des tribunats militaires. Selon le rang dans la société romaine dépendront le ou les tribunats accessibles. Ainsi, il n'est pas étonnant que le recrutement vienne cibler les plus hautes sphères sociales de la société, notamment l'ordre équestre. La seconde inégalité que met en exergue l'étude de l'auteur réside dans l'existence d'un recrutement militaire géographiquement ciblé. De fait, certaines régions et provinces de I'Empire se voient favorisées au détriment d'autres. Troisième et non des moindres, l'existence d'une classification hiérarchique ascendante au cœur même des tribunats légionnaires. Les mentions épigraphiques ne semblent pas mettre au même niveau toutes les légions, préférant certaines plutôt que d'autres selon les avantages qu'elles peuvent apporter à I'Empire.

D'une tout autre nature, le discours de Sophie Trierweiler se concentre sur la terminologie du droit juridique homérique par le biais des termes de dikê et de themis. Se basant sur les poèmes homériques, l'auteure souligne le lien étroit qu'il peut exister entre ces deux concepts tout en relevant leurs différences et les possibles rapports de sujétions qu'ils révèlent. Ces concepts au double sens désignent à la fois deux déesses, Thémis épouse de Zeus et Dikê dont elle est la mère, mais aussi deux champs d'application du droit strictement distincts. Si I'une, la themis, vient essentiellement désigner les comportements acceptés ou proscrits pour le maintien de l'ordre et de l'équilibre dans les sociétés humaines, l'autre, la dikê incarne la justice, la sentence ou encore le règlement public. Ainsi, l'étroitesse de ces deux notions laisse vite place à l'image d'un véritable droit hiérarchisé où Zeus apparaît au point culminant de la société homérique.

Si I'intérêt des approches statistiques dans I'analyse typologique et hiérarchique des sites archéologiques n'est plus à démontrer, c'est notamment grâce aux travaux précurseurs des projets Archaeomedes et ArchaeDyn. La contribution de François Favory vient ici résumer près d'une trentaine d'années de recherches sur la question de la structuration et I'organisation de I'habitat antique. Trouvant ses racines au début des années 1980, « l'expérience Archaeomedes » a permis d'aborder 
les dynamiques de I'habitat antique du sud-est de la France méditerranéenne tout en mettant en œuvre une terminologie et une chaîne opératoire descriptive commune. Comme le rappelle I'auteur, I'importance d'une recherche de consensus méthodologiques appropriés permet d'innover tout en traitant de manière optimale un grand nombre de données. L'auteur revient à de nombreuses reprises sur le terme d'expérience, mais explique qu'à cette époque les méthodes et les outils employés devaient encore démontrer tout leur potentiel. Potentiel qui a rapidement été développé, en permettant de proposer une classification des établissements selon différentes variables par le biais d'outils, de méthodes et de démarches novateurs souvent considérés, parfois encore aujourd'hui, comme avant-gardistes. C'est grâce à l'élaboration de ce socle méthodologique, mais aussi des résultats obtenus, que les programmes Archaeomedes et ArchaeDyn résonnent encore aujourd'hui dans de nombreux travaux portant sur l'analyse organisationnelle des établissements.
La question de la hiérarchie dans les sociétés actuelles a été abordée par Louise Beyrand à travers la question du patrimoine culturel immatériel du Cambodge. Malheureusement, sa disparition tragique ne lui a pas permis d'achever ses travaux. Nous tenons à rendre hommage dans le premier chapitre à notre collaboratrice, une jeune chercheuse qui nous a quittés trop tôt.

Si la grande hétérogénéité des contributions peut paraître un frein à la réflexion sur le thème proposé de la hiérarchie et de la hiérarchisation, ces regards croisés, à travers le prisme d'horizons disciplinaires plus ou moins lointains - chaque discipline venant avec ses a priori et ses méthodes, ont permis d'enrichir les discussions sur les différentes façons d'appréhender ces questions. C'est par la confrontation d'idées, de méthodes et de démarches parfois presque perçues comme antagonistes que peuvent naître des discours originaux tout en permettant au chercheur de prendre du recul sur sa propre approche. Puisse le présent dossier thématique témoigner de l'efficacité de cette démarche ! 